PROCEEDINGS OF THE

AMERICAN MATHEMATICAL SOCIETY

Volume 128, Number 8 , Pages $2405-2411$

S 0002-9939(99)05308-3

Article electronically published on November 29, 1999

\title{
FINITE GENERATION PROPERTIES FOR FUCHSIAN GROUP VON NEUMANN ALGEBRAS TENSOR $B(H)$
}

\author{
FLORIN RĂDULESCU
}

(Communicated by David R. Larson)

\begin{abstract}
We prove that the algebra $\mathcal{A}=\mathcal{L}\left(F_{N}\right) \otimes B(H), F_{N}$ a free group with finitely many generators, contains a subnormal operator $J$ such that the linear span of the set $\left\{\left(J^{*}\right)^{n} J^{m} \mid n, m=0,1,2, \ldots\right\}$ is weakly dense in $\mathcal{A}$. This is the analogue for the $I I_{\infty}$ factor $\mathcal{L}\left(F_{N}\right) \otimes B(H), N$ finite, of a well known fact about the unilateral shift $S$ on a Hilbert space $K$ : the linear span of all the monomials $\left(S^{*}\right)^{n} S^{m}$ is weakly dense in $B(K)$.

We also show that for a suitable space $H^{2}$ of square summable analytic functions, if $P$ is the projection from the Hilbert space $L^{2}$ of all square summable functions onto $H^{2}$ and $M_{-}$is the unbounded operator of multiplication by $\bar{j}$ on $L^{2}$, then the (unbounded) operator $P M_{\bar{j}}(I-P$ ) is nonzero (with nonzero domain).
\end{abstract}

In this paper we prove the existence of finite systems $\mathcal{G}$ of generators, with special properties, for the von Neumann algebras $\mathcal{L}(\Gamma) \bar{\otimes} B(H)$, obtained by tensoring the group von Neumann algebra of a fuchsian group $\Gamma$ with the bounded linear operators on an infinite dimensional, separable, Hilbert space $H$. The results show that the linear span of ordered monomials that are products of powers of adjoints of elements in $\mathcal{G}$ with powers of elements in $\mathcal{G}$ is weakly dense in the algebra. In addition the systems of generators are a commuting family, consisting of subnormal operators.

If $\Gamma=P S L(2, \mathbb{Z})$, then there exists a single subnormal operator $J$, in the algebra $\mathcal{A}=\mathcal{L}(P S L(2, \mathbb{Z})) \otimes B(H)$ (which by the results in $([\overline{\mathrm{Dyk}}],[\mathrm{Ra} 2])$ is isomorphic to $\mathcal{L}\left(F_{N}\right) \otimes B(H), F_{N}$ a free group with finitely many generators), such that linear span of the set $\left\{\left(J^{*}\right)^{n} J^{m} \mid n, m=0,1,2, \ldots\right\}$ is weakly dense in $\mathcal{A}$. This is the analogue for the $I I_{\infty}$ factor $\mathcal{L}\left(F_{N}\right) \otimes B(H), N$ finite, of a well known fact about the unilateral shift $S$ on a Hilbert space $K$ : the linear span of all the monomials $\left(S^{*}\right)^{n} S^{m}$ is weakly dense in $B(K)$. The operator $J$ comes from a Toeplitz operator having as symbol Dedekind's modular invariant function $j$ and acting on a suitable Hilbert space of analytic functions.

The construction in this paper may also be used to provide examples for Toeplitz operators with unbounded symbol, with unexpected behaviour, and non-closability for multiplication operators, with bounded symbol, in Sobolev type Hilbert spaces

Received by the editors September 22, 1998.

1991 Mathematics Subject Classification. Primary 46L35; Secondary 46L37, 46L57, 81S99, $11 \mathrm{~F} 99$.

The author's research was supported in part by the grant DMS 9622911 from the National Science Foundation. The author is a member of the Institute of Mathematics, Romanian Academy, Bucharest. 
associated to differential operators commuting with the laplacian. More precisely we will show that for a suitable space $H^{2}$ of square summable analytic functions, if $P$ is the projection from the Hilbert space $L^{2}$ of all square summable functions onto $H^{2}$ and $M_{\bar{j}}$ is the unbounded operator of multiplication by $\bar{j}$ on $L^{2}$, then the (unbounded) operator $P M_{\bar{j}}(I-P)$ is nonzero (with nonzero domain). This is in contrast to what happens when $\phi$ is a bounded analytic function in $H^{2}$; then $P M_{\bar{\phi}}(I-P)=0$.

In proving this result we will rely on the construction outlined in Ra3 on the equivariant Berezin quantization of the upper half-plane and on a remarkable result of Murray and von Neumann about the algebra of unbounded operators affiliated to a type $I I_{1}$ factor $(\underline{\mathrm{MvN}})$.

\section{TOEPLitZ OPERATORS With UNBOUNDED SYMBOL}

In this section we outline the construction of the Toeplitz operator with unbounded and antianalytic symbol the modular function $j$. This operator is not closable and this will allow us to construct the non zero, unbounded operator of the form $P M_{\bar{j}}(I-P)$.

Recall some of the notations that were used in [Ra3]. Let $H_{t}$ be the Hilbert space of square summable, analytic functions on $\mathbb{H}$, with respect to the measure $\mathrm{d} \nu_{t}=(\operatorname{Im} z)^{t-2} \mathrm{~d} \bar{z} \mathrm{~d} z$. In $[\overline{\mathrm{Pu}}],[\mathrm{Sa}]$ it was proven that there exists a one parameter family $\left(\pi_{t}\right)_{t>1}$, of irreducible, projective unitary representation of $\operatorname{PSL}(2, \mathbb{R})$ on $H_{t}$ that extends the analytic discrete series of representations for $\operatorname{PSL}(2, \mathbb{R})$. Moreover the methods in [GHJ], along with the trace formula in $[\mathrm{Pu}$, were used in ( $\mathrm{Ra3}]$ ) to show that for any fuchsian group $\Gamma$, the von Neumann algebra $\left\{\pi_{t}(\Gamma)\right\}^{\prime \prime}$ is a type $I I$ factor acting on $H_{t}$. The Murray-von Neumann dimension for this algebra acting on $H_{t}$ is proportional to $t$ and the covolume of $\Gamma$. In fact, the commutant algebras $\mathcal{A}_{t}=\left\{\pi_{t}(\Gamma)\right\}$ " are isomorphic to the twisted group von Neumann algebra $\mathcal{L}\left(\Gamma, \sigma^{t}\right)_{(t-1) / \text { covolume } \Gamma}$ (or to $\mathcal{L}\left(\Gamma, \sigma^{t}\right) \otimes B(H)$ if the covolume of $\Gamma$ is infinite), with $\sigma^{t}$ the cocycle corresponding to the projective representation $\pi_{t}$.

As shown in [GHJ, automorphic forms $g$ (for $\Gamma$ ) of weight $k$, where $k$ is a strictly positive integer, correspond to bounded intertwining operators $S_{g}^{t}$ from $H_{t}$ into $H_{t+k} . S_{g}^{t}$ is the multiplication operator with $g$ acting on $H_{t}$. The intertwining property means that

$$
\pi_{t+k}(\gamma) S_{g}^{t}=S_{g}^{t} \pi_{t}(\gamma), \gamma \in \Gamma
$$

Let $f$ be any measurable, bounded $\Gamma$-invariant function on $\mathbb{H}$. Let $M_{f}^{t}$ be the multiplication operator by $f$ on $H_{t}$ and let $P_{t}$ be the projection operator from bounded square summable functions on $\mathbb{H}$ onto $H_{t}$. The Toeplitz operator $T_{f}^{t}=$ $P_{t} M_{f}^{t} P_{t}$ clearly commutes with $\left\{\pi_{t}(\Gamma)\right\}$ and hence $T_{f}^{t}$ belongs to $\mathcal{A}_{t}$.

Also, we consider in this paper Toeplitz operators, not necessarily bounded, having as symbols $\Gamma$ invariant, analytic functions on $\mathbb{H}$ (i.e. automorphic forms of order 0 ). Unfortunately, as we show below, though affiliated with the commutant algebras, such an operator is not densely defined, nor is it closable. One possible symbol function, for $\Gamma=P S L(2, \mathbb{Z})$, is the classical modular invariant function $j=\frac{\Delta}{G_{4}^{3}}$.

Lemma 1. Let $\Gamma$ be a fuchsian group, of finite covolume, and let $j$ be an analytic, $\Gamma$-invariant function on $\mathbb{H}$. Assume that $j=\frac{a}{b}$ is the quotient of two automorphic forms for $\Gamma$ having the same weight $m$. Assume that the functions 
$z \rightarrow|a(z)|^{2}(\operatorname{Im} z)^{m-2}, z \rightarrow|b(z)|^{2}(\operatorname{Im} z)^{m-2}$, are bounded. Also we assume that $a, b$ have not all their zeroes and poles in common.

For a bounded, measurable, $\Gamma$ invariant function $f$ on $\mathbb{H}$, let $T_{f}^{t}$ be the Toeplitz operator on $H_{t}$ with symbol $f$. Clearly, $T_{f}^{t}$ belongs to the commutant $\mathcal{A}_{t}=$ $\left\{\pi_{t}(P S L(2, \mathbb{Z})\}^{\prime}\right.$ and the set of all such operators is a weakly dense subspace of the Hilbert space $L^{2}\left(\mathcal{A}_{t}, \tau\right)$, associated with the trace $\tau$ on $\mathcal{A}_{t}$ (see [Ra1]).

Then the operator $T_{f}^{t} \rightarrow T_{\bar{j} f}^{t}$, whose domain is a dense subset of $L^{2}\left(\mathcal{A}_{t}, \tau\right)$, is non closable, for any $t>1$.

Moreover the same holds true if we replace $\bar{j}$ by $|j|^{2}$ or by $\frac{\bar{j}}{j}$.

Proof. The hypothesis shows that the bounded (see [GHJ]) operators $S_{a}^{t}, S_{b}^{t}$ on $H_{t}$ into $H_{t+k}$, defined by by multiplication with $a$ and respectively $b$, have nonequal projections onto the closure of their ranges. Let $c$ be any other automorphic form $\Gamma$ having the same order as $a, b$. Let $\Psi$ be the operator in the statement. Then for any $\Gamma$-invariant, bounded, measurable function $h$ we will have that

$$
\Psi\left(\left(S_{a}^{t}\right)^{*} T_{h}^{t+k} S_{c}^{t}\right)=\left(S_{b}^{t}\right)^{*} T_{h}^{t+k} S_{c}^{t} .
$$

This holds because obviously $\left(S_{a}^{t}\right)^{*} T_{h}^{t+k} S_{c}^{t}=T_{\bar{a} h c}^{t}$ and $\left(S_{b}^{t}\right)^{*} T_{h}^{t+k} S_{c}^{t}=T_{\bar{b} h c}^{t}$.

Let $e_{a}$ be the projections onto the range of $S_{a}^{t}$, which is a subspace of $H_{t+k}$. As the set of all $T_{h}^{t+k}$, when $h$ runs through the bounded, measurable, $\Gamma$-equivariant functions on $\mathbb{H}$, is weakly dense in the commutant $\mathcal{A}_{t+k}$, it follows that for any $x$ in $\mathcal{A}_{t+k}$ we may find a sequence of such functions $h_{n}$ on $\mathbb{H}$ such that $T_{h_{n}}^{t+k}$ converges weakly to $\left(1-e_{a}\right) x$ and hence $\left(S_{a}^{t}\right)^{*} T_{h_{n}}^{t+k} S_{c}^{t}$ converges weakly to 0 . Then $\left(S_{b}^{t}\right)^{*} T_{h_{n}}^{t+k} S_{c}^{t}$ converges weakly to $\left(S_{b}^{t}\right)^{*}\left(1-e_{a}\right) x S_{c}^{t}$. If $\Psi$ were closable, then it would follow that $\left(S_{b}^{t}\right)^{*}\left(1-e_{a}\right) x S_{c}^{t}$ is zero for all $x$ in $\mathcal{A}_{t}$. Since $x$ is arbitrary, it follows that $\left(S_{b}^{t}\right)^{*}\left(1-e_{a}\right)=0$.

Because the operators $S_{a}^{t}, S_{b}^{t}$ have non equal projections onto the closure of their ranges, while those projections have the same trace (as both operators are injective), this is impossible. This completes the proof for $\bar{j}$.

The statement for the other two functions is proved in a similar way: multiplication by $|j|^{2}$ maps $\left(S_{a}^{t}\right)^{*} T_{h}^{t+k} S_{a}^{t}$ into $\left(S_{b}^{t}\right)^{*} T_{h}^{t+k} S_{b}^{t}$ (and this is a positive map) and multiplication by $\frac{\bar{j}}{j}$ maps $\left(S_{a}^{t}\right)^{*} T_{h}^{t+k} S_{b}^{t}$ into $\left(S_{b}^{t}\right)^{*} T_{h}^{t+k} S_{a}^{t}$.

Remark 2. With the notations in the above statement let $F$ be a fundamental domain for the action of $\Gamma$ on $\mathbb{H}$. Let $B_{t}=B_{t}(\Delta)$ be the Berezin operator defined by

$$
B_{t} f(z)=\int_{\mathbb{H}} f(w)\left[\frac{(\operatorname{Im} z)(\operatorname{Im} w)}{\bar{z}-w}\right]^{t-2}(\operatorname{Im} w)^{-2} \mathrm{~d} \bar{w} \mathrm{~d} w, \quad z \in \mathbb{H} .
$$

Note that the operator $B_{t}$ commutes with the invariant laplacian ( $\left.\mathrm{Be}\right]$ ) on $L^{2}\left(F,(\operatorname{Im} w)^{-2} \mathrm{~d} \bar{w} \mathrm{~d} w\right)$. Then $L^{2}\left(\mathcal{A}_{t}, \tau\right)$ is identified with the the completion of a dense subset of $L^{2}\left(F,(\operatorname{Im} w)^{-2} \mathrm{~d} \bar{w} \mathrm{~d} w\right)$ with the scalar product

$$
\langle f, g\rangle=\left(B_{t}(\Delta) f, g\right)_{L^{2}(F)} .
$$

With this scalar product, the operator of multiplication by $\bar{j}$ on a dense subspace of $L^{2}\left(F,(\operatorname{Im} w)^{-2} \mathrm{~d} \bar{w} \mathrm{~d} w\right)$ is non closable. Moreover the same holds true if we replace $\bar{j}$ by $|j|^{2}$ or by $\frac{\bar{j}}{j}$. 
The following corollary shows that one of the properties that is valid for Toeplitz operators with bounded, antianalytic symbol fails for unbounded symbols (see also [JS], [Saf] $)$.

Corollary 3. Let $\Gamma$ be a fuchsian group of finite covolume, and let $j=\frac{a}{b}$ be an analytic, $\Gamma$ invariant function on $\mathbb{H}$ as above. Let $P_{t}$ be the projection from $L^{2}\left(\mathbb{H},(\operatorname{Im} z)^{t-2} d \bar{z} d z\right)$ onto $H_{t}=H^{2}\left(\mathbb{H},(\operatorname{Im} z)^{t-2} d \bar{z} d z\right)$. Let $M_{\bar{j}}$ be the multiplication operator with $\bar{j}$ which is defined on a dense subset of the Hilbert space $L^{2}\left(\mathbb{H},(\operatorname{Im} z)^{t-2} d \bar{z} d z\right)$.

Then $P_{t} M_{\bar{j}}\left(1-P_{t}\right)$ is nonzero (in particular has nonzero domain).

Proof. Assume the contrary. Let $h, h_{1}$ be any $\Gamma$-invariant, bounded, measurable, real valued functions $h, h_{1}$ on $\mathbb{H}$, such that the operators $T_{h}^{t}, T_{h_{1}}^{t}$ are injective. Also, assume that the supports of $h, h_{1}$ are so that the functions $\bar{j} h, \bar{j} h_{1}$ are bounded.

Let $Z_{h}, Z_{h_{1}}$ be the closable operators ( $\left.\underline{\mathrm{MvN}}\right]$ ), defined by

$$
Z_{h}=T_{\bar{j} h}^{t}\left(T_{h}^{t}\right)^{-1}, Z_{h_{1}}=T_{\bar{j} h_{1}}^{t}\left(T_{h_{1}}^{t}\right)^{-1} .
$$

Our assumption implies that

$$
T_{\bar{j} h}^{t} \zeta=T_{\bar{j} h_{1}}^{t} \zeta_{1} \text { whenever } T_{h}^{t} \zeta=T_{h_{1}}^{t} \zeta_{1} .
$$

This implies that the closable, unbounded operators $Z_{h}, Z_{h_{1}}$ coincide on a densely defined core and hence that they are equal (again by $[\mathrm{MvN}]$ ).

Hence there exists a unique, closed operator, affiliated with $\mathcal{A}_{t}=\left\{\pi_{t}(\Gamma)\right\}^{\prime}$ such that for any real valued, bounded, measurable functions $h$, with $T_{h}^{t}$ invertible, we have (by $[\mathrm{MvN}]$ )

$$
Z T_{h}^{t}=T_{\bar{j} h}^{t}
$$

But this is impossible by the previous statement.

Corollary 4. With the notations in the previous statement, the same conclusion holds if $j$ is replaced by $h \circ j$, where $h$ is any univalent entire function $h$.

Proof. By examining the above argument, we see that in fact we proved that if $K$ is any compact subset of the interior of $F$ such that $j$ is bounded when restricted to $K$ and $\chi_{K}$ is the characteristic function of $K$, then

$$
P_{t} M_{\bar{j}}\left[\left(\mathrm{Id}-P_{t}\right) \wedge \chi_{K}\right] \neq 0 .
$$

Now assume that the (bounded) linear operator $M_{\overline{f \circ j}}$ would have the property that

$$
M_{\overline{f \circ j}}\left[\left(\left(\mathrm{Id}-P_{t}\right) \wedge \chi_{K}\right)\left(H_{t}\right)\right] \subseteq\left(\mathrm{Id}-P_{t}\right)\left(H_{t}\right) .
$$

It would then follow that $M_{\overline{f \circ j}}$ would also have the property that

$$
M_{\overline{f \circ j}}\left[\left(\left(\mathrm{Id}-P_{t}\right) \wedge \chi_{K}\right)\left(H_{t}\right)\right] \subseteq\left(\left(\mathrm{Id}-P_{t}\right) \wedge \chi_{K}\right)\left(H_{t}\right) .
$$

Since $f$ is univalent, the same would then hold true about $j$ instead of $f \circ j$, and this we know to be false.

Questions. (i). Is the above statement true if one drops the univalence condition on $f$ ?

(ii). If $t>24, \Gamma=P S L(2, \mathbb{Z})$ and $j=\frac{\Delta}{G_{4}^{3}}=\frac{\Delta^{2}}{G_{4}^{3} \Delta}$, then it is clear that the domain of $M_{\bar{j}}$ intersects $H_{t}$ nontrivially. Does this hold for smaller values of $t$ ? 


\section{Generation properties}

In this section we construct the system of generators for algebras of the form $\mathcal{L}(\Gamma) \otimes B(H)$ where $\Gamma$ is a fuchsian group with special properties. We will then specialize to the case $\Gamma=P S L(2, \mathbb{Z})$ and show one can reduce the system of generators to a single generator in this case.

Assume first that $\Gamma$ is a fuchsian group (of infinite covolume). Assume that $H^{\infty}(\mathbb{H} / \Gamma)$ has a sufficiently rich structure so that there are a finite number of bounded analytic functions that separate points on $\mathbb{H} / \Gamma$. In this case, by using the methods developed in [Ra3] we can show that $\mathcal{L}(\Gamma) \otimes B(K)$ has a set of generators with the properties in the following proposition.

Proposition 5. Let $\Gamma$ be a fuchsian group such that $H^{\infty}(\mathbb{H} / \Gamma)$ contains functions $h_{1}, \ldots, h_{k}$ that separate the points on $\mathbb{H}$. (For examples of such groups see [St].) Let $K$ be an infinite dimensional Hilbert space. Then there exist commuting, bounded subnormal operators $Z_{1}, \ldots, Z_{k}$ in $\mathcal{B}=\mathcal{L}(\Gamma) \otimes B(K)$ such that

$$
\mathcal{B}={\overline{\operatorname{Sp~}\left\{\left(Z_{i}^{n}\right)^{*} Z_{j}^{m} \mid n, m \in \mathbb{N}, i, j=1,2, \ldots, k\right\}}}^{\text {weak }} .
$$

Proof. We consider the algebra $\mathcal{B}=\left\{\pi_{t}(\Gamma)\right\}^{\prime \prime}$. Since $\Gamma$ has infinite covolume, it follows that $\mathcal{B}$ is isomorphic to $\mathcal{L}(\Gamma) \otimes B(K)$. Let $Z_{i}=T_{h_{i}}^{t}, i=1, \ldots, k$. Let $c$ be an arbitrary element in $\mathcal{B} \cap L^{1}(\mathcal{B}, \tau)$, with zero kernel. Assume that $a \in \mathcal{B}$ is orthogonal to the following subspace of $L^{1}(\mathcal{B}, \tau)$ :

$$
{\overline{\operatorname{Sp~}\left\{c\left(Z_{i}^{n}\right)^{*} Z_{j}^{m} \mid n, m \in \mathbb{N}, i=1, \ldots, k\right\}}}^{\text {weak }} .
$$

We use the notations in [Ra3]. Let $(\hat{a c})(\bar{z}, z), z \in \mathbb{H}$, be the Berezin symbol of $a c \in \mathcal{B} \cap L^{1}(\mathcal{B}, \tau)$. The trace formula in [Ra3] shows that

$$
\int_{F}(\hat{a c})(\bar{z}, z) \overline{h_{i}^{n}(z)} h_{j}(z)(\operatorname{Im} z)^{t-2} \mathrm{~d} \bar{z} \mathrm{~d} z=0 .
$$

Hence $a c=0$ and hence $a=0$. Consequently

$$
\text { Sp }\left\{c\left(Z_{i}^{n}\right)^{*} Z_{j}^{m} \mid n, m \in \mathbb{N}, i=1, \ldots, k\right\}
$$

is a weakly dense subspace of $L^{1}(\mathcal{B}, \tau)$ and consequently, since $c$ has zero kernel, it follows that

$$
{\overline{\mathrm{Sp}\left\{\left(Z_{i}^{n}\right)^{*} Z_{j}^{m} \mid n, m \in \mathbb{N}, i, j=1, \ldots, k\right\}}}^{\text {weak }}=\mathcal{B} \text {. }
$$

Proposition 6. Let $E$ be an open, $\Gamma$-invariant, subset of $\mathbb{H}$, such that $j$ is bounded on $E$. Let $H_{t}(E)$ be the subspace of $L^{2}\left(\mathbb{H}, \nu_{t}\right)$, consisting of square summable, analytic functions on $E$ (that are extended with 0 outside $E$ ).

Then $H_{t}(E)$ is $\Gamma$-invariant, with respect to the unitary representation $\pi_{t}$ of $P S L(2, \mathbb{R})$ (restricted to $\Gamma)$, on $L^{2}\left(\mathbb{H}, \nu_{t}\right)$. Moreover, $H_{t}(E)$ is an infinite, Hilbert, left module over $\mathcal{L}(\Gamma)$ (and a left submodule, over $\mathcal{L}(\Gamma)$, of $L^{2}\left(\mathbb{H}, \nu_{t}\right)$ ).

Let $Z$ be the Toeplitz operator on $H_{t}(E)$ with symbol $\left.j\right|_{E}$. Then the commutant of $\pi_{t}(\Gamma)$ in $B\left(H_{t}(E)\right)$ is the weak closure of the linear span of the set $\left\{\left(Z^{*}\right)^{n} Z^{m} \mid n, m=\right.$ $0,1,2, \ldots\}$.

Proof. Clearly $H_{t}(E)$ is a submodule of $L^{2}\left(\mathbb{H}, \nu_{t}\right)$ over $\mathcal{L}(\Gamma)$. Let $k_{E}=k_{E}^{t}$ be the reproducing kernel for $H_{t}(E)$ and let $F(E)$ be a fundamental domain for $\Gamma$ acting on $E$. Then the Murray-von Neumann dimension of $H_{t}(E)$ as a left module over 
$\mathcal{L}(\Gamma)$ is equal to $\int_{F(E)} k_{E}(z, z) \mathrm{d} \nu_{t}(z)$. This is obviously infinite, as no subnormal operator (that is not normal) can live in a type $I I_{1}$ factor. (I owe this last remark to Ken Dykema.)

Moreover, as in $\mathrm{Be}$, if $A$ is an operator acting on $H_{t}(E)$, we define its Berezin kernel (with respect $H_{t}(E)$ ) to be

$$
k_{A}^{E}(z, \zeta)=\frac{\left\langle A e_{z}^{E, t}, e_{\zeta}^{E, t}\right\rangle}{\left\langle e_{z}^{E, t}, e_{\zeta}^{E, t}\right\rangle}, z, \zeta \in E
$$

where $e_{z}^{E, t}$, for $z$ in $E$, is the evaluation vector in $H_{t}(E)$ at $z$.

If $A$ is an operator in $B\left(H_{t}(E)\right.$ that commutes with the action of $\Gamma$, and if $A$ is in the trace ideal of the commutant algebra (which is a type $I I_{\infty}$ factor), then the trace of $A$ in the commutant algebra is given (as in [Ra3]) by the formula

$$
\int_{F(E)} k_{A}^{E}(z, z) \frac{k_{E}^{t}(z, z)}{(\operatorname{Im} z)^{t}} \mathrm{~d} \nu_{0}(z)
$$

Note that the factor $\frac{k_{E}^{t}(z, z)}{\operatorname{Im} z)^{t}}$ is $\Gamma$-invariant. This is the trace on the commutant of $\Gamma$ on $L^{2}\left(\mathbb{H}, \nu_{t}\right)$ that is normalized by giving value $\frac{t-1}{\pi}$ to $H^{2}\left(\mathbb{H}, \nu_{t}\right)$.

Let $f$ be a bounded $\Gamma$-invariant function on $E$ and let $T_{f, E}^{t}$ be the Toeplitz operator on $H_{t}(E)$ with symbol $f$. As in [Ra3] (see also [Be]), the formula for the trace in the commutant algebra of $A T_{f, E}^{t}$ is

$$
\int_{F(E)} k_{A}^{E}(z, z) f(z) \frac{k_{E}^{t}(z, z)}{(\operatorname{Im} z)^{t}} \mathrm{~d} \nu_{0}(z) .
$$

Hence, if an operator $A$, commuting with $\Gamma$ and in the trace ideal of the commutant algebra, is orthogonal (with respect to the trace on the commutant) on all Toeplitz operators on $H_{t}(E)$, with $\Gamma$-equivariant symbol, then it follows that $A=0$. Hence the linear span of all Toeplitz operators, with bounded, $\Gamma$-equivariant symbols, is weakly dense in the commutant. Since the symbol of $\left(Z^{*}\right)^{n} Z^{m}$ is $\left(\left.\bar{j}\right|_{E}\right)^{n}\left(\left.j\right|_{E}\right)^{m}$, the statement follows by the Stone-Weierstrass theorem.

As a corollary of the previous proposition we get our main result

Theorem 7. In the algebra $\mathcal{A}=\mathcal{L}\left(P S L(2, \mathbb{Z}) \otimes B(H)=\mathcal{L}\left(F_{N}\right) \otimes B(H)\right.$, where $N$ is finite, there exists a bounded, subnormal operator $Z$, such that $\mathcal{A}$ is the weak closure of linear span of the set $\left\{\left(Z^{*}\right)^{n} Z^{m} \mid n, m=0,1,2, \ldots\right\}$.

\section{ACKNOWLEDGEMENT}

The author is indebted to K. Dykema and P. de la Harpe for very usefu comments.

\section{REFERENCES}

[Be] F. A. Berezin, Quantization in complex symmetric spaces, Math USSR Izvestija, 9(1975), 341-379. MR 58:22691

[Co] J. B. Conway, Subnormal Operators, Research Notes in Mathematics v. 51, Pitman Advanced Publ. Prog., Boston, 1982. MR 83i:47030

[Dyk] K. Dykema, Free products of hyperfinite von Neumann algebras and free dimension, Duke Math. J. 69, 97-119 (1993). MR 93m:46071]

[GHJ] F. Goodman, P. de la Harpe, V.F.R. Jones, Coxeter Graphs and Towers of Algebras, Springer Verlag, New York, Berlin, Heidelberg, 1989. MR 91c:46082 
[JS] Jan Janas, Stochel, J , Unbounded Toeplitz operators in the Segal-Bargmann space. II. MR 95m:47040

[MvN] F. J. Murray, J. von Neumann, On ring of Operators,IV, Annals of Mathematics, 44 (1943), 716-808. [MR 5:101a]

$[\mathrm{Pu}] \quad$ L. Pukanszki, The Plancherel formula for the universal covering group of $\operatorname{PSL}(2, \mathrm{R})$, Math Annalen, 156 (1964), 96-143. MR 30:1215

[Pi] G. Pisier, Espaces de Banach quantiques: une introduction à la théorie des espaces des operateurs, Journèe Annuelle, Soc. Math. France, 1994. MR 98e:46019

[Ra1] F, Rădulescu,On the von Neumann Algebra of Toeplitz Operators with Automorphic Symbol, in Subfactors, Proceedings of the Taniguchi Symposium on Operator Algebras, edts. H. Araki, Y. Kawahigashi, H. Kosaki, World Scientific, Singapore-New Jersey, 1994. MR 96j:47024

[Ra2] F. Rădulescu, Random matrices, amalgamated free products and subfactors in free group factors of noninteger index, Inv. Math.115, pp. 347-389, (1994). MR 95c:46102

[Ra3] F. Rădulescu, The arithmetic Hecke operators and Berezin Quantization, Comptes Rendu Acad. Sci. Paris, Serie I. Mathématique 322 (1996), no. 6, 541-546. MR 97i:46112

[Ra4] F. Rădulescu, The $\Gamma$ invariant form of the Berezin quantization of the upper halfplane (Preprint 1995, Memoirs A. M. S., 1998. MR 98j:46080

[Sa] P. Sally, Analytic Continuation of the Irreducible Unitary Representations of the Universal Covering Group, Memoirs A. M. S., 1968. MR 38:3380

[St] Stout, E. L., On some algebras of analytic functions on finite open Riemann surfaces. Math. Z., 92, 1966 366-379. MR 34:358

[Saf] F. H. Szafraniec, Unbounded subnormal operators, Bull. Iranian Math. Soc. 17 (1990), 67-79. MR 91f:47035

[Vo] D. Voiculescu, Circular and semicircular systems and free product factors, in Operator Algebras, Unitary Representations, Enveloping algebras and Invariant Theory, Prog. Math., 92, pp. 45-60, Boston: Birkhauser 1990. MR 92e:46124

Department of Mathematics, The University of Iowa, Iowa City, Iowa 52246

E-mail address: radulesc@math.uiowa.edu 